\title{
Frequency adjectives and assertions about event types
}

\author{
Berit Gehrke \& Louise McNally \\ Universitat Pompeu Fabra
}

\section{Introduction}

In this paper we present a semantics for a little-studied and problematic class of adjectives, the so-called frequency adjectives (FAs), such as occasional and rare. FAs have been associated with three different readings, which we descriptively label the internal reading, the generic reading, and the adverbial reading, following the literature (Stump 1981, Larson 1998, Zimmermann 2003, Schäfer 2007).

The INTERNAL READING is typically found when the FA modifies a deverbal, participant-denoting noun. On this reading, [FA N] can roughly be paraphrased as "N who/that V's/is V'd FA-ly", as exemplified in (1).

(1) That claim was made by an occasional sailor.

= That claim was made by someone who sails occasionally.

The internal reading is so-called because the semantic effect of the FA is within the DP in which it appears, in contrast to the adverbial reading, to be described shortly.

The GENERIC READING is illustrated in (2). As the name suggests, sentences manifesting the generic reading typically express generalizations about the sort of individual described by their subject nominal. In many examples of the generic reading, for example as illustrated in (2a), the [FA N] nominal is paraphrasable as an event description, even if the head noun does not denote an event, and the FA serves to describe the event in question. However, this paraphrase is not always available, as shown in (2b) (examples from Stump 1981).

(2) a. An occasional beer is good for you.

$=$ Drinking a beer occasionally is good for you.

b. An occasional beer tastes good on a hot day. $\neq$ Drinking a beer occasionally tastes good on a hot day.

Finally, the ADVERBIAL READING shown in (3), first observed in Bolinger (1967) and no doubt the most puzzling reading, can be paraphrased by prefixing the FA as a sentential adverbial to the complete sentence.

(3) The/an occasional sailor strolled by.

= Occasionally, a sailor strolled by.

We gratefully acknowledge the comments we received from the audiences at SALT XIX and the GLOW 32 Semantics Workshop on Modes of Composition. This work was supported by grant HUM2007-60599 from the Spanish Ministry of Education and Science and by an ICREA Acadèmia award from the Institut Català de Recerca i Estudis Avançats. 
This reading thus contrasts sharply with the other readings insofar as the FA seems to scope outside of the nominal it occurs in. No such interpretation is possible for the FA on the internal reading, as mentioned above, nor for the generic reading.

The above examples pose two sorts of semantic composition problems. First, we must account for the seeming ability of the FA in (3) to escape from within the DP and take sentence level scope, given that DPs are typically scope islands. Second, we must account for the fact that FAs, which intuitively characterize events, combine not only with nouns that directly describe events (such as agentive nouns like sailor in (1)) or involve indirect reference to events (such as deverbal nouns like destruction and non-derived nouns referring to something like an event, e.g. trip), but also with nouns that do not make reference to an event by themselves, such as beer in (2). Since these problems do not appear with the internal reading, this paper will mainly focus on the generic and the adverbial readings.

In addition, an adequate analysis of the semantics of FAs faces the challenge of accounting for these different readings while maintaining a unified semantics for the adjective and avoiding ad hoc assumptions about its syntax. Specifically, we want to account for the adverbial reading without treating the FA as a determiner on that reading, as proposed in Stump (1981), Larson (1998), and Zimmermann (2003). To do so, we will take as a starting point Schäfer (2007), the analysis that comes closest to meeting this challenge, point out its problems, and argue that these problems can be solved if sentences like (3) are treated as assertions about event types, rather than event tokens, as is usually assumed on event semantic analyses.

The paper is structured as follows. After presenting the data in more detail, we outline two previous types of accounts: the determiner analysis of the adverbial reading (Stump 1981, Larson 1998, Zimmermann 2003), and Schäfer's (2007) unified analysis of the generic and adverbial readings, and the problems they face. We then present our own proposal and its advantages and conclude with some unresolved issues and general comments.

\section{Additional data}

We have two goals in presenting additional details concerning the data. The first is to bring together the major observations about FAs that have been made in previous works and to add some of our own; we consider such a review useful considering the small literature on FAs and the fact that previous authors have not always agreed as to what these facts are. The second, and more important goal, is to make clear why we think the data do not immediately call for a special analysis of the adverbial reading but rather are compatible with a unified approach to the semantics of FAs.

\subsection{Lexically-related restrictions on the readings of FAs}

The availability of the different readings for FAs is sensitive to three different factors which we mention briefly here, though we must leave a detailed analysis of their 
influence for future research. The first factor has to do with the specific semantics of the FA. FAs can be subclassified along two parameters. One is whether the FA specifies a fixed periodicity, such as daily, or a variant, contextually-determined periodicity, such as occasional. Within this latter group, we must distinguish those specifying relative frequency (e.g. frequent) from those specifying relative infrequency (e.g. occasional). The second factor is the semantics of the modified noun. FAs combine with three different types of nouns, namely eventuality-denoting nouns, such as visit, swimming, discussion, participant-denoting nouns, such as recipient, sailor, employee, and other nouns, such as beer, car, chair. Throughout the paper, we will use the terms EVENT NOUNS, PARTICIPANT NOUNS, and SORTAL NOUNS, respectively, to refer to these classes. Finally, the third factor is the semantics of the predicate to which the nominal containing the FA appears as an argument.

The restrictions that we and others have identified on the various readings of FAs include the following. First, only participant nouns yield THE INTERNAL READING (Schäfer 2007) (4a,b).
a. A frequent sailor won the regatta. = one who sails frequently
b. A frequent recipient of awards took the Rotary Club prize again. $=$ one who frequently receives awards
c. A frequent visit $\neq$ a particular visit which happens frequently
d. A frequent letter $\neq$ a particular letter which Vs/is V'd frequently

On the internal reading the nominal describes an individual whose identity is stable across the events that support the applicability of the FA. This is why this reading is blocked with event nouns unless those nouns can be understood as describing types of events - whose identity is stable across token events -, as in (5).

(5) The Linguistics faculty meeting is a frequent meeting.

Furthermore, the FA describes the frequency with which the nominal description holds of the individual it describes. This fact makes the internal reading pragmatically implausible for sortal nouns.

A second restriction is related to THE GENERIC READING. All kinds of FA-noun combinations can yield the generic reading, if the NP is an argument to a generic predicate (in the sense of Carlson 1977); consider (6).
a. A yearly / an occasional / a frequent check-up is essential.
b. A yearly / an occasional / a frequent visitor is great to have.
c. A daily / an occasional / a frequent cup of coffee is good for you.

With stage-level predicates, on the other hand, the FA is not or at least is not obviously generically interpreted, as (7) shows.

(7) a. ??An occasional cup of coffee has left a stain on the table. (Stump 1981)

b. The Premier Division-based Scotland side were only beaten, in fact, by a goal of almost tragic proportions, conceded when an infrequent error was characteristically punished by Riedle.

(from the $B N C$ ) 
There are two standard approaches to indefinite generics. Arguably the more common approach is to assume that the indefinite lacks its own quantificational force, and that it gets this force by ending up in the restriction of a generic operator contributed by e.g. tense (see for example Heim 1982). Another, much less popular approach, advocated in e.g. Müller-Reichau (2006) is to treat the indefinite as denoting a kind or an existential quantifier over kinds, extending Carlson's (1977) analysis of bare plurals. ${ }^{1}$ Observe that in order to get the right interpretation for the sentences in (6), some version of the latter option seems inevitable, since generic quantification does not yield the right results, either with generic quantificat! ion over objects (8a) or over events (8b).

a. (Gen $x: x$ is a cup of coffee and you occasionally drink $x)[x$ is good for you]

b. (Gen $e: e$ is a cup-of-coffee-drinking and $e$ is occasional)[ $e$ is good for you]

Generic quantification over objects in (8a) cannot be right since a token cup of coffee cannot be drunk more than once. Generic quantification over events in (8b), on the other hand, will not work because a token event cannot be occasional.

A sketch of a logical representation involving generic quantification that might work if we could get the details right is given in (9).

(9) (Gen $x$ : $x$ realizes the "cup of coffee drunk occasionally" kind) [ $x$ is good for you]

However, since it is not at all obvious how to get those details right, we opt instead for the kind approach in our account, since it yields the right readings directly.

Finally, there are various restrictions on the availability of THE ADVERBIAL READING. For starters, only event nouns systematically yield the adverbial reading with the three kinds of adjectives (10).

The group held a weekly / an occasional / a frequent discussion session.

With non-event nouns the picture is more complicated. Although Zimmermann (2003) (following Larson 1998) claims, contra Stump (1981), that the adverbial reading is only possible with infrequency adjectives (occasional, infrequent, rare, and $o d d$ ), our intuitions coincide with Stump's (see also Moltmann 1997, Schäfer 2007). However, it does seem to be the case that only relative infrequency adjectives systematically yield the adverbial reading with singular non-event nouns. To get the adverbial reading with relative frequency adjectives (e.g. frequent), a plural noun is needed (11).

a. ??She wrote a frequent letter to her mother.

b. She wrote frequent letters to her mother.

\footnotetext{
${ }^{1}$ One motivation for such an analysis is the acceptability of indefinite kind terms which do not refer to subkinds in examples like This morning Fred invented a pumpkin crusher, an example attributed to Bart Geurts.
} 
$=$ Frequently, she wrote letters to her mother.

c. ??She baked a frequent batch of cookies.

d. She baked frequent batches of cookies.

$=$ Frequently, she baked batches of cookies.

With both fixed frequency and relative frequency adjectives, the adverbial reading is subject to additional, poorly understood restrictions. One example of an additional restriction is given in (12). Though further data collection is necessary, the contrast between (11) and (12) and some additional examples we have collected suggests that when an FA other than occasional modifies a sortal noun, the adverbial reading is possible only if the noun is a complement to a verb of creation.

a. They saw a daily train on their way to school.

$\neq$ Every day they saw a train on their way to school.

b. ??They saw frequent trains on their way to school.

c. ??They read frequent/daily books.

These data raise the question whether the number difference calls for a different treatment of the adverbial reading with the two kinds of variant FAs. Larson (1998) and Zimmermann (2003) think so, but we, like Schäfer (2007), are not convinced. Furthermore, we have to ask whether the contrasts between event nouns and the other nouns call for the adverbial reading with the latter to be dealt with in a different way. Stump (1981) treated them uniformly, whereas Zimmermann (2003) assumes they are different. We will follow Stump on this point.

We now turn to another sort of restriction on the use of FAs.

\subsection{Determiner-related restrictions on the readings of FAs}

There are also restrictions with respect to the determiner that can appear with FAnoun combinations on the generic and adverbial readings. In this respect these readings contrast with the internal reading, which is available with all kinds of determiners; see (13).

(13) $\mathrm{A} /$ Some/One/That/Each frequent sailor I know owns his own boat.

Specifically, both the generic and the adverbial reading are only possible with the (in)definite articles and semantically bleached possessives as illustrated in (14) and (15), respectively.

(14) a. An/the/your occasional beer is good for you.

b. ??Each occasional beer is good for you.

c. ??Two occasional beers are good for you.

(15) a. We saw an/the/your occasional car on the road.

b. ??We saw each occasional car on the road.

c. ??We saw two/many occasional cars on the road. 
Although Stump (1981) disagrees on this point, claiming instead that the generic reading allows a full variety of determiners, upon closer inspection his examples (which include (16a)) turn out to differ in an important way from those in (13).

a. No daily news program can match this one.

b. Every daily cup of coffee is good for you.

Specifically, while the quantification in (13) is over token sailors, (16b), to the extent that it is acceptable, only can be interpreted as quantifying universally over kinds of cups of coffee, not over token cups. In our research we have found examples like this one to be more representative than those resembling (16a). In the case of (16a), daily news program is a well-established expression for a particular kind of program, and we would therefore argue that the compositional semantics for daily news program is no longer transparent in the way that the semantics of daily cup of coffee is. The fact that an unrestricted range of determiners is possible only when quantifying over kinds, while in other cases only the (in)definite articles and bleached possessives are possible, strongly suggests that the FA on the generic and adverbial readings is part of a DP that necessarily denotes a kind or a quantifier over kinds. If this ! were not the case, we would expect DPs such as the subject of (16b) to be able to denote quantifiers over token cups of coffee. This finding resonates with the observations made above concerning the analysis of the genericity in (6) and plays an important role in motivating the analysis we develop below, on which FAs will serve as modifiers of kind descriptions but not of token-entity descriptions.

To conclude this section, though the facts are complex, the differences between the generic and adverbial readings are smaller than previously claimed, removing one possible argument for giving the adverbial reading a separate analysis. Moreover, we have seen some reasons for positing that nominals containing FAs on both the generic and adverbial readings must denote descriptions of kinds, rather than descriptions of token entities, again an argument for pursuing a unified analysis of these readings.

\section{Previous analyses of FAs}

There have been two previous approaches to FAs, and to the adverbial reading in particular: the "determiner" analysis (Stump 1981, Larson 1998, Zimmermann 2003), on which the adverbial reading is given a separate syntactic and semantic treatment from the internal and generic readings; and the unified analysis of all three readings, exemplified in Schäfer (2007). We will begin with the determiner analysis, with the goal of showing (1) that the arguments for a special treatment of the adverbial reading are not convincing, and (2) that there are facts that the determiner analyses cannot account for. 


\subsection{The determiner analysis of the adverbial reading}

To illustrate the determiner analysis, we take Zimmermann (2003) (who closely follows Larson 1998) as representative. Zimmermann proposes that the adverbial reading results when the FA syntactically incorporates into the determiner, which forms with it a complex pluractional quantifier INFREQ over event-individual pairs. His proposed syntax and semantics for the resulting complex determiner for a sentence like (17) is provided in (18). ${ }^{2}$

An/the occasional sailor strolled by.

a. Syntax (Zimmermann 2003: 271, minor details modified):

$\left[\mathrm{IP}\left[\mathrm{QP}\left[\mathrm{Q} \text { the/an+occasional }{ }_{1}\right]\left[\mathrm{NP} t_{1} \text { sailor }\right]\right]_{2}\left[\mathrm{VP}_{2} t_{2}\right.\right.$ strolled by $\left.]\right]$

b. Semantics (Zimmermann 2003: 272, notation slightly modified):

(i) $[$ an/the occasional $] \Rightarrow[\operatorname{INFREQ}\langle e, x\rangle]$

(ii) $[\mathrm{INFREQ}\langle e, x\rangle]=$ $\lambda Q \lambda S . \exists\langle e, x\rangle[$ part-of $(e, e *) \wedge Q(x)]$ $\left[S(e, x) \wedge \forall\left\langle e^{\prime}, x^{\prime}\right\rangle,\left\langle e^{\prime \prime}, x^{\prime \prime}\right\rangle\left[S\left(e^{\prime}, x^{\prime}\right) \wedge S\left(e^{\prime \prime}, x^{\prime \prime}\right) \wedge Q\left(x^{\prime}\right) \wedge Q\left(x^{\prime \prime}\right)\right]\right.$ $\left[\left(e^{\prime}=e^{\prime \prime}\right) \vee\left(e^{\prime} \neq e^{\prime \prime} \wedge \exists t\left[\right.\right.\right.$ between $\left.\left.\left.\left.\left(t, \tau\left(e^{\prime}\right), \tau\left(e^{\prime \prime}\right)\right)\right]\right)\right]\right]$ (where $e *=$ a contextually given event, $\tau(e)=$ the temporal trace of $e$ )

A complete semantic representation for (17) is given in (19a); (19b) provides an informal paraphrase (Zimmermann 2003: 273).

a. $\quad \exists\langle e, x\rangle[\operatorname{part}-o f(e, e *) \wedge \operatorname{sailor}(x)]$

$\left[\operatorname{stroll} \_b y(x, e) \wedge \forall\left\langle e^{\prime}, x^{\prime}\right\rangle,\left\langle e^{\prime \prime}, x^{\prime \prime}\right\rangle\left[\operatorname{stroll} \_b y\left(e^{\prime}, x^{\prime}\right) \wedge \operatorname{stroll} \_b y\left(e^{\prime \prime}, x^{\prime \prime}\right) \wedge\right.\right.$ sailor $\left.\left(x^{\prime}\right) \wedge \operatorname{sailor}\left(x^{\prime \prime}\right)\right]$

$\left.\left[\left(e^{\prime}=e^{\prime \prime}\right) \vee\left(e^{\prime} \neq e^{\prime \prime} \wedge \exists t\left[\operatorname{between}\left(t, \tau\left(e^{\prime}\right), \tau\left(e^{\prime \prime}\right)\right)\right]\right)\right]\right]$

b. There are some pairs $\langle e, x\rangle$, where $e$ is a part of a contextually given event $e *$, and $x$ a sailor, such that $e$ is a strolling-by of $x$, and any two strolling-by events of a sailor occur at separate points in time.

This analysis correctly predicts that on the adverbial reading the FA scopes over the variable corresponding to the nominal (and, indeed, over the entire sentence). It also accounts for the fact that only $a$, the, and bleached possessives combine with FAs on the adverbial reading: Zimmermann proposes that these determiners lack semantic content, and that complex quantifier formation is only possible if the determiner lacks such content. Finally, it accounts for various kinds of "unadjectival" behavior of FAs that were identified in Stump (1981) and which played a role in motivating his version of the determiner analysis. We now turn to this latter behavior with the goal of showing that, in fact, that behavior does not call for a determiner analysis of FAs.

\footnotetext{
${ }^{2}$ The "between" condition in the analysis is intended to guarantee that the events making the sentence true are distributed over time and not clumped together.
} 


\subsubsection{The putative un-adjectival nature of FAs}

The first un-adjectival property identified by Stump (1981) is that the FA on the adverbial reading must appear external to other adjectives. If it appears closer to the head than other adjectival material, as in (20a), only the internal reading is possible; (20a) lacks the adverbial reading found in (20b).
a. A well-dressed occasional sailor strolled by.
b. $\neq$ An occasional well-dressed sailor strolled by.
(Stump 1981: 248)

However, there are other adjectives, such as other, which have to appear external to other adjectival expressions, as (21) shows.

*Two well-dressed other sailors strolled by.

Nevertheless, this restriction has not motivated a determiner analysis of other or similarly behaving adjectives.

A second un-adjectival property Stump identifies is the failure of the FA to conjoin with other adjectives; see (22).

$$
\text { *An occasional and well-dressed sailor strolled by. }
$$

(Stump 1981: 249)

However, this is not always the case. (23) shows that with event nouns, such coordinations are attested.

(23) There are occasions when, because of outside atmospheric conditions, an occasional and brief down draft will occur within the chimney and fire box... (http://www.patentstorm.us/patents/6298844/description.html)

Though the reader might argue that (23) is not a valid counterexample because it involves an event noun, and there might be significant differences in the interpretation of FAs with event vs. non-event nouns, the reader should also observe that (23) is a clear example of the adverbial reading: it can be paraphrased as "Occasionally, a brief down draft will occur within the chimney and fire box...." This fact suggests that it must be possible for the FA to provide the illusion of wide scope while still functioning as an adjective; and if that is so for event nouns, we might be optimistic that it is the case for other kinds of nouns as well.

A third argument put forward by Stump for the claim that FAs are not proper adjectives is that they cannot appear in the antecedent of a common noun anaphor, as shown in (24).

$$
\begin{aligned}
& \text { a. John saw an occasional sailor, and Bill saw one, too. (Stump 1981: } \\
& \text { 248) } \\
& \text { b. } \quad \neq \text { Occasionally John saw a sailor, and occasionally Bill saw one, too. }
\end{aligned}
$$

However, once again this is not a good reason to claim that FAs are not proper adjectives, since other indisputable adjectives, such as different, manifest similar behavior, as (25) illustrates. 
a. ?A well-dressed different sailor strolled by.

b. A different and well-dressed sailor strolled by. [cf. (23)]

c. Mary saw a sailor. John saw a different sailor, and Bill saw one $(\neq \mathrm{a}$ different sailor), too.

To conclude, while the data show that FAs on the adverbial reading are different from adjectives that express typical properties of individuals (such as physical properties), there are other clear examples of adjectives that manifest the same behavior that FAs do on the adverbial reading. We therefore conclude that these facts do not force a determiner analysis of FAs.

\subsubsection{Problems for the determiner analysis}

Beyond the facts in (20)-(24), which we have just shown not to be convincing motivation, there is no independent motivation for the determiner-like syntax and semantics for FAs. Moreover, there are problems for the determiner analysis. For example, it is not obvious how to extend the semantics in (18-b) to the other readings. In addition, there is no trigger for the movement of the FA in (18-a) that licenses the adverbial reading. A third problem is that if the FA is interpreted as a quantifier, it is not clear why it must be accompanied by a real determiner, as shown by the the ungrammaticality of (26).

*Occasional sailor strolled by.

Finally, the determiner analysis makes some incorrect predictions concerning scope. For example, there is no non-stipulative account for the failure of the FA to scope over the main verb in examples like (27), whose only interpretation is that in (27b).

(27) a. Idling beside the propped-open kitchen window he registers the occasional car swishing past, three stories below. (from the $B N C$ )

b. = He registers the cars that swish past, which they do occasionally.

Zimmermann discusses similar cases in German and argues that they do not involve INFREQ because of independently motivated restrictions on quantifier raising $(\mathrm{QR})$ from object position in German. However, this explanation cannot be plausibly extended to English because such restrictions on QR are not motivated for English. Yet this use of occasional greatly resembles the adverbial reading, scope notwithstanding, and it would be desirable to account for it under the same analysis.

Since the determiner analysis poses more problems than it solves and, furthermore, since it does not provide a uniform account of FAs under all readings, we will not pursue it further and instead turn to another account in the literature.

\subsection{A first attempt at a unified analysis: Schäfer (2007)}

The basic idea of Schäfer's (2007) account is that FAs on all three readings contribute the same core semantics, namely frequency quantification over events, al- 
though there are small variations in the compositional details. In the following, we discuss only the generic and adverbial readings.

Several key assumptions underly Schäfer's account. First, he assumes that there are event kinds, event individuals, and event stages in the ontology. Our account shares this assumption and in section 4 we will provide additional motivation for event kinds, in particular. Schäfer specifically proposes that FAs with event nominals on all three readings modify an event variable associated with that nominal. FAs with non-event nominals, on the other hand, are argued to involve coercion of the noun the FA modifies (e.g. a cup of coffee) so that it is interpreted as a contextually determined event description (e.g. drinking a cup of coffee). In the following notations, these and all other contextually valued variables are prefixed with $\uparrow$.

Schäfer's semantics for FAs builds on the idea that they introduce probability functions that determine the likelihood of there being a realization of a particular kind of event involving a particular kind of individual in a particular role for a particular interval which is checked for the realization of this proposition. The details are somewhat technical and not crucial to our discussion of his analysis, so we will present them here quickly and with little comment, referring the reader to his work for fuller explanation. All FAs are characterized in terms of a 4-place probability function $p\left(e_{k}\right)\left(x_{k}\right)(\Theta)(i)$, where $e_{k}, x_{k}$, and $\Theta$ supply parameters for the proposition that there is a realization of an event kind $e_{k}$ involving an individual kind $x_{k}$ in role $\Theta$, which he formulates as in (28).

$$
\exists e_{s} \cdot \exists x_{s} \cdot \mathbf{R}\left(e_{k}\right)\left(e_{s}\right) \wedge \Theta\left(x_{s}\right)\left(e_{s}\right) .
$$

The variable $i$ in the probability function is the "checking interval" for this proposition, which can either be fixed, for fixed periodicity FAs (e.g. daily for daily), or determined pragmatically, for FAs of variant periodicity, as calculated by an additional function $\mathbf{i}$, defined in (29).

$\mathbf{i}\left(e_{k}\right)\left(x_{k}\right)(\Theta)={ }_{\text {def }}$ the prototypical checking interval for event kind $e_{k}$, with object kind $x_{k}$ in role $\Theta$ (abbreviated below as $\mathbf{I}$, with parameters assumed to be supplied)

The value of $p\left(e_{k}\right)\left(x_{k}\right)(\Theta)(i)$ is a probability range $[m, n]$. It will be low for FAs like occasionally (specifically, Schäfer suggests 30-60\%, or $[.3, .6]$ ), high for those like frequent, and approximating certainty (e.g. $[.98,1])$ for FAs expressing a fixed periodicity, like daily.

\subsubsection{The generic and adverbial readings}

With this background, we now turn to Schäfer's analysis of the generic and adverbials readings. ${ }^{3}$ Schäfer proposes that, under the generic reading, an FA like occa-

\footnotetext{
${ }^{3}$ Though space precludes discussing the internal reading, for completeness we reproduce his analysis of occasional walker (Schäfer 2007: 563) in (i), in slightly simplified notation. Note that in order for this analysis to work, Schäfer assumes a "bracketing paradox" to allow the FA to modify the verb root directly in walker. Also note that the FA is subscripted to distinguish its translation on
} 
sional introduces an event kind variable and an event kind description $\uparrow E$, which effectively coerces the nominal containing the FA into a description of a kind of event with a low frequency of realization (Schäfer 2007: 564). In the example in (30b) this event kind would be a drinking or other action involving a cup of coffee.

\section{a. occasional 2 :}

$$
\lambda P \lambda e_{k} . \exists x_{k} . \uparrow E\left(e_{k}\right) \wedge P\left(x_{k}\right) \wedge p\left(e_{k}\right)\left(x_{k}\right)(\uparrow \Theta)(\mathbf{I})=[.3, .6]
$$

b. occasional 2 cup of coffee:

$$
\lambda e_{k} \cdot \exists x_{k} \cdot \uparrow E\left(e_{k}\right) \wedge \text { cup_of_coffee }\left(x_{k}\right) \wedge p\left(e_{k}\right)\left(x_{k}\right)(\uparrow \Theta)(\mathbf{I})=[.3, .6]
$$

For the adverbial reading, Schäfer switches the binding of the event kind and individual kind variables, so that the resulting nominal denotes a description of an individual kind rather than an event kind; the latter is existentially quantified over. When this nominal is combined with the main verb, stages of the individual kind are going to be involved in stages of the event kind with the relevant probability of realization frequency (Schäfer 2007: 564f.). However, what will this event kind $\uparrow E\left(e_{k}\right)$ in which the sailor stages in (31b) occasionally participate actually be? Schäfer observes that something must guarantee that $\uparrow E\left(e_{k}\right)$ be identified with the description contributed by the main verb. That is, for (3), it must be an instance of the kind of sailors who occasionally stroll by that serves as the subject and not, for example, the kind of sailors who occasionally take a swim.

$$
\begin{array}{ll}
\text { a. } & \text { occasional }_{3}: \\
& \lambda P \lambda x_{k} \cdot \exists e_{k} \cdot \uparrow E\left(e_{k}\right) \wedge P\left(x_{k}\right) \wedge p\left(e_{k}\right)\left(x_{k}\right)(\uparrow \Theta)(\mathbf{I})=[.3, .6] \\
\text { b. } & \text { occasional }_{3} \text { sailor: } \\
& \lambda x_{k} \cdot \exists e_{k} \cdot \uparrow E\left(e_{k}\right) \wedge \operatorname{sailor}\left(x_{k}\right) \wedge p\left(e_{k}\right)\left(x_{k}\right)(\uparrow \Theta)(\mathbf{I})=[.3, .6]
\end{array}
$$

However, Schäfer does not propose any specific mechanism to guarantee this identification between $\uparrow E\left(e_{k}\right)$ and the main verb description, nor does he compute the semantics for a full DP or sentence containing (32b).

\subsubsection{Pros and cons of the analysis}

One advantage of Schäfer's analysis is that it does not postulate any unmotivated syntactic category or movement for the FA; rather, it makes use of a standard semantics for the adjective as property of properties. However, his account also faces various problems. First, the semantics for the FA is not entirely unified: on the

the internal reading from its translation on the other readings; see the text for additional comments.

$$
\begin{aligned}
& \text { a. } \quad \text { walk } \Rightarrow \lambda e_{k} \cdot \text { walk }\left(e_{k}\right) \\
& \text { b. } \quad \text { occasional }_{1} \Rightarrow \lambda E . \lambda e_{k} \cdot E\left(e_{k}\right) \wedge p\left(e_{k}\right)\left(\uparrow x_{k}\right)(\Theta)(\mathbf{I})=[.3, .6]
\end{aligned}
$$

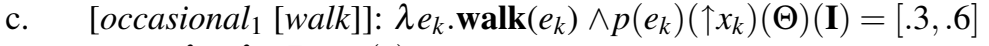

$$
\begin{aligned}
& \text { d. } \quad-e r \Rightarrow \lambda E . \lambda x \cdot \exists e_{k} . E(e) \wedge \operatorname{agent}\left(x, e_{k}\right) \\
& \text { e. } \quad\left[\text { occasional }_{1}[\text { walk }] \text { er }\right]:\left(\lambda E \cdot \lambda y \cdot \exists e_{k} \cdot E\left(e_{k}\right) \wedge \operatorname{agent}\left(y, e_{k}\right)\right) \\
& \left(\lambda e_{k} \cdot \mathbf{w a l k}\left(e_{k} \wedge p\left(e_{k}\right)\left(\uparrow x_{k}\right)(\uparrow \Theta)(\mathbf{I})=[.3, .6]\right)\right. \\
& =\lambda y \cdot \exists e_{k} \cdot \mathbf{w a l k}\left(e_{k}\right) \wedge p\left(e_{k}\right)\left(\uparrow x_{k}\right)(\uparrow \Theta)(\mathbf{I})=[.3, .6] \wedge \operatorname{agent}\left(y, e_{k}\right)
\end{aligned}
$$


generic reading it denotes a function from properties of individuals to properties of event kinds, while on the adverbial reading it denotes a function from properties of individuals to properties of individual kinds. Second, it is not clear that a probabilistic semantics will guarantee the appropriate number of event realizations: this semantics only guarantees that there is a certain probability of such realizations existing. Third, it is not clear why $\uparrow E$ should get its value from context on the generic reading but have to be identified with the main predicate description on the adverbial reading and, as mentioned, it! is not clear how to ensure the latter identification. Fourth, the analysis of the generic reading fails to account for one of the subcases of this reading mentioned at the beginning of the paper, namely the one on which the nominal does not denote an event description; recall (2-b), repeated in (32).

An occasional cup of coffee tastes good.

Schäfer's analysis, as it stands, incorrectly predicts that the sentence means that drinking a cup of coffee occasionally tastes good.

Finally, under the standard assumption that sentences introduce existential quantification over event tokens, Schäfer's analysis of the adverbial reading produces problematic results. To see why, we provide what we think would be the simplest and most uncontroversial derivation for the rest of (3) with the indefinite article, which, for illustration, we treat as an existential quantifier, though this is not crucial (see also below):

a. $\quad$ occasional sailor: $\lambda x_{k} \exists e_{k}\left[\uparrow E\left(e_{k}\right) \wedge\right.$ sailor $\left(x_{k}\right) \wedge\left[p\left(e_{k}\right)\left(x_{k}\right)(\uparrow \Theta)(\mathbf{I})=\right.$ $[.3, .6]]]$

b. an occasional sailor:

$\lambda P \exists x_{k} \exists e_{k}\left[\uparrow E\left(e_{k}\right) \wedge \operatorname{sailor}\left(x_{k}\right)\right.$

$\left.\wedge\left[p\left(e_{k}\right)\left(x_{k}\right)(\uparrow \Theta)(\mathbf{I})=[.3, .6]\right] \wedge P\left(e, x_{k}\right)\right]$

c. $\quad$ strolled by: $\lambda x \exists e\left[\mathbf{s t r o l l e d} \_b \mathbf{b y}(e, x)\right]$

d. An occasional sailor strolled by:

$\exists x_{k} \exists e_{k}\left[\uparrow E\left(e_{k}\right) \wedge\right.$ sailor $\left(x_{k}\right) \wedge\left[p\left(e_{k}\right)\left(x_{k}\right)(\uparrow \Theta)(\mathbf{I})=[.3, .6]\right]$

$\wedge \exists e$.strolled_by $\left.\left(x_{k}, e\right)\right]$

Assume that $\uparrow E$ is identified as "strolling by". The sentence will then assert that a token event of strolling by was performed by that kind of sailor who participates in strolling by events occasionally. But this is not equivalent to the adverbial reading or indeed any reading of the sentence.

Although Schäfer's analysis faces problems, the idea that event and individual kinds are involved is an important insight and will serve as a starting point for our own proposal, which we now present.

\section{The proposal}

We will follow Schäfer in having the FA combine with a nominal that describes a kind, and also in giving the FA the function of imposing conditions on the re- 
alizations of the nominal kind, without making use of the problematic probability function. Our key innovation involves analyzing sentences like (2) and (3) as existential quantifications not over event tokens but rather over event types or kinds (for present purposes we use these latter terms interchangeably).

Event types are natural to expect if we assume that events form a subsort in our ontology of (token) individuals (Reichenbach 1947, Davidson 1967, Parsons 1990), that kinds or types form another subsort in that ontology (Carlson 1977), and that, as a rule, any token in the ontology should be the realization of some type in that ontology. In addition, our use of event types has an antecedent in e.g. the Situation Semantics notion of event type (Barwise and Perry 1983), though the formal details are quite different. Empirical arguments for event types as an ontological category have been made in e.g. Landman and Morzycki (2003) and Ginzburg (2005).

We further assume that nouns can systematically be interpreted as properties of kinds, in addition to denoting properties of token entities (see e.g. McNally and Boleda 2004, Müller-Reichau 2006: for defense of this idea) and that verbs likewise can denote relations involving event types, in addition to relations involving event tokens. Argument positions of predicates denoting properties of event types can be filled either by kind-level or token-level arguments, as illustrated in (34). ${ }^{4}$

strolled by: $\lambda x_{\alpha} \lambda e_{\alpha}\left[\operatorname{strolled} \_b y\left(e_{\alpha}, x_{\alpha}\right)\right]$, where $\alpha$ ranges over both kinds and tokens.

In more familiar examples, the existence of a kind at some index $i$ (specifying world, location and interval, for instance) simply depends on finding one token realization of that kind at $i$. The kinds described using FAs are crucially different: Their existence at an index $i$ depends on not merely the existence of one token realization of the kind, but on the existence of a set of token realizations, possibly with additional properties - e.g., distribution with a certain spatial or temporal periodicity (see Stump 1981).

The translation we propose for occasional is given in (35a), which has the satisfaction conditions in (35b), where distribution is a function that yields the distribution of a set of individuals at a given index and low indicates that the value qualifies as low in the context.

$$
\begin{array}{ll}
\text { a. } & \text { occasional: } \lambda P \lambda x_{k}\left[(\operatorname{occasional}(P))\left(x_{k}\right)\right] \\
\text { b. } & \forall P, x_{k}, i\left[(\operatorname{occasional}(P))\left(x_{k}\right) \text { at } i \leftrightarrow\right. \\
& \left.\left[P\left(x_{k}\right) \wedge \text { distribution }\left(\left\{y: \mathbf{R}\left(y, x_{k}\right)\right\}, i\right)=d_{\text {low }}\right]\right]
\end{array}
$$

For the combination of the FA with a noun and a determiner, we treat the indefinite article in the spirit of Kamp (1981) or Heim (1982). We analyse the definite article as a maximality operator, yielding the unique maximally general kind described by the nominal. These assumptions yield the representations in (36).

\footnotetext{
${ }^{4}$ In other words, we think it is as conceptually sound to talk of an event type involving Popeye as it is to talk of one involving sailors in general.
} 


$$
\begin{aligned}
& \text { a. an occasional } N:(\operatorname{occasional}(\mathbf{N}))\left(x_{k}\right) \\
& \text { b. the occasional } N: \operatorname{MAX} x_{k}\left[(\mathbf{o c c a s i o n a l}(\mathbf{N}))\left(x_{k}\right)\right]
\end{aligned}
$$

The analysis of the adverbial reading with the indefinite nominal is represented in (37), where we assume that existential closure binds off all unbound individual and event variables.

$$
\begin{aligned}
& \text { a. } \quad \text { An occasional sailor strolled by. } \\
& \text { b. } \quad \exists x_{k}, e_{k}\left[(\text { occasional }(\operatorname{sailor}))\left(x_{k}\right) \wedge \operatorname{strolled} \_b y\left(e_{k}, x_{k}\right)\right]
\end{aligned}
$$

The adverbial reading with the definite nominal is correspondingly as in (38).

$$
\begin{aligned}
& \text { a. The occasional sailor strolled by. } \\
& \text { b. } \quad \exists e_{k}\left[\mathbf{s t r o l l e d} \_\mathbf{b y}\left(e_{k}, \mathbf{M A X} x_{k}\left[(\mathbf{o c c a s i o n a l}(\operatorname{sailor}))\left(x_{k}\right)\right]\right)\right]
\end{aligned}
$$

What is of course of utmost importance here is the satisfaction conditions for these representations. While we will not assume that all kinds of predications over event types necessarily entail the existence of token events that realize the type, any assertion that an event type exists at an index $i$ will entail the existence of at least one token event at $i$ that supports the existence assertion. When the predicate describes an event in which token entities necessarily participate, this will entail the existence of individual token entities corresponding to any kinds in the logical representation for the event type, as in (39a) (where $P$ is restricted to ranging over just these kinds of predicates); if the event kind involves an individual kind whose realization requires a set of individuals, as in the case of sentences containing FAs, it is natural to expect that the realization of the event kind will depend on the existence of sets of event tokens; see (39b) (we assume $x_{k}$ to range over the just those kinds realized by sets of tokens).

$$
\begin{array}{ll}
\text { a. } & \forall e_{k}, x_{k}, P, i\left[P\left(e_{k}, x_{k}\right) \text { at } i \leftrightarrow \exists e, x\left[\mathbf{R}\left(e, e_{k}\right) \wedge \mathbf{R}\left(x, x_{k}\right) \wedge P(e, x) \text { at } i\right]\right] \\
\text { b. } & \forall e_{k}, x_{k}, P, X, i\left[X=\left\{x: \mathbf{R}\left(x, x_{k}\right) \text { at } i\right\} \rightarrow\left[P\left(e_{k}, x_{k}\right) \text { at } i \leftrightarrow\right.\right. \\
& \left.\left.\left[\forall x \in X \exists e\left[\mathbf{R}\left(e, e_{k}\right) \wedge P(e, x) \text { at } i\right]\right]\right]\right]
\end{array}
$$

Now consider the generic reading. First note that, unlike Schäfer, we do not assume that the generic reading always involves coercion of the nominal description to an event description, given the existence of examples like the one in (2-b). Rather, the interpretation of the nominal on the generic reading depends on the semantics of the main predicate, as is in fact quite generally the case with nominals, whether they contain an FA or not, and whether they are generically interpreted or not. Consider the familiar sort of example in (40), where the predicate enjoy is what determines that the object is interpreted as if it denoted an event involving the book in question, rather than the book itself.

(40) The kids enjoyed the book.

We posit that whatever theory accounts for the interpretation of the book in (40) should be used to account for the subcase of the generic reading on which the nominal seems to describe an event rather than an object. One such recent theory is 
proposed in Asher (2007), to which we refer the reader for details. Asher's analysis is technically complex, but essentially reduces to legitimating, via a set of general coercion rules, the substitution of an event description in the place of a nominal description in the representation of a clause, whenever the lexical semantics of the predicate to which that nominal serves as an argument so permits or requires. On this view, the nominal on the generic reading will have only one basic representation: exactly the same one it has on the adverbial reading. However, as a result of coercion, the description of a nominal such as the occasional beer may turn out to be essentially as Schäfer proposed. Thus, borrowing Schäfer's notation $\uparrow E$ for a contextually-specified (here, understood as coerced) event description, the representation for (41a) and (42a) will be as in (41b) and (42b), respectively.

a. The occasional beer tastes good.

b. $\exists e_{k}\left[\operatorname{tastes}\left(e_{k}, \operatorname{MAX} x_{k}\left[(\right.\right.\right.$ occasional $($ beer $\left.\left.\left.))\left(x_{k}\right)\right], \operatorname{good}\right)\right]$

a. The occasional beer (i.e. drinking an occasional beer) is good for you.

b. $\quad \exists e_{k} \exists e_{k}^{\prime}\left[\operatorname{good}\right.$ for $\_$you $\left(e_{k}, e_{k}^{\prime}\right)$ $\wedge \uparrow E\left(e_{k}^{\prime}, \mathbf{M A X} x_{k}\left[(\right.\right.$ occasional $($ beer $\left.\left.\left.))\left(x_{k}\right)\right]\right)\right]$

The essential difference between the generic and the adverbial readings has to do with the conditions that must be satisfied in order for token events of the kinds in (41) and (42) to exist. We assume that the properties taste good and be good for you can be meaningfully used to characterize kinds. Thus, (41) can be paraphrased as asserting that the kind of thing which is beer and any given realization of which is a set of token entities distributed over a particular frequency will taste good. In an important class of cases, if such a predicate can be ascribed to a kind, it will hold for a significant number of relevant realizations of the kind. Thus, if the occasional beer kind tastes good, a significant number of relevant realizations of that kind that is, sets of token beers in a particular temporal distribution - will taste good. Parallel reasoning will guarantee the correct satisfaction conditions for (42b).

Our proposal has the following advantages. In contrast to what is assumed on the determiner analysis, we do not posit any unmotivated syntactic movement or syntactic category for the FA; the latter simply denotes a property of properties. The alleged un-adjectival behavior identified by Stump is expected given the lexical semantics of the FA: since it does not contribute to the basic descriptive content of the kind eventually denoted by the nominal but rather simply imposes conditions on the kind's realization, it is unsurprising that it must take scope over that description, and that it does not participate in common noun phrase anaphora or coordinate with adjectives that do contribute to the descriptive content of the kind. Furthermore we provide a unified account of the adverbial and generic readings of FAs, which we believe should be straightfowardly extendable to the internal reading and to examples like (27-a), though space precludes demonstrating this here. Our account also correctly predicts that FAs only combine with a limited set of determiners, without requiring the stipulation that these articles are contentless: The articles that appear with FAs are precisely those that are compatible with basic kind terms. Indeed, our analysis makes a further correct prediction: bare plurals containing FAs 
are also acceptable, as (43) shows.

She wrote occasional letters to her mother.

\section{Some remaining issues}

Our account solves the major problems we see with previous accounts but leaves some issues unresolved, although it must be said that previous accounts do not fare any better. For example, the analysis has nothing to say at this point about the lexical restrictions on the combinatorial possibilities of different kinds of FAs with different kinds of nouns and the respective restrictions on the availability of particular readings, mentioned in section 2. It is also not clear what explains the different restrictions on the distributions of fixed and variant FAs.

Another unsolved question is why FAs resist combining with mass nonevent nouns, as demonstrated in (44).
a. There was occasional trouble.
b. ??Occasional beer is good for you.
c. ??Occasional water dropped from the ceiling.
d. Occasional drops of water fell from the ceiling.

We can only make the preliminary observation that the nominal seems to have to be able to individuate the token events in question: compare (44) with (45a-b).
a. ??They baked occasional cookies.
b. They baked occasional batches of cookies.

There are additional puzzles related to the determiner restrictions on the generic and adverbial readings. For example, contrary to what has been claimed in the literature, plural some and $a$ few sometimes seem acceptable with both readings:

a. A few/some occasional beers are good for you. $=$ It is good for you to have a few/some beers on occasion.

b. She does still leave a few occasional "fool's raisins" just outside of her box. (http://www.barnhartgallery.com/houserabbits.htm) = Occasionally she leaves a few "fool's raisins" outside her box.

In addition, non-deictic demonstratives seem possible with the generic but not the adverbial reading:

a. That occasional beer is good for you.

b. ??We saw that occasional car on the road.

A third unexplained fact is that, on the generic reading, the definite article with frequent yields only an anaphoric reading, not the "indefinite" reading typically associated with that reading (see (48a)); and the definite article never seems to 
appear with frequent on the adverbial reading (see (48b)).

a. The frequent cups of coffee are good for you.

b. John wrote the frequent letters to his mother. $\neq$ Frequently, John wrote the letters to his mother.

In contrast, FAs like odd and rare prefer the definite article and resist the indefinite article, as shown in (49).

a. ??A(n)/The odd/rare visitor is nice to have.

b. We saw ??a(n)/the odd/rare car.

Thus, though the range of determiners licensed with FAs is indeed limited, it is slightly broader than generally claimed.

All of these puzzles raise the questions of whether there are important semantic differences in the subclasses of FAs, and if so, what they are, but we must leave these questions for future research.

\section{Conclusion}

Though a small lexical class, FAs present a set of interesting and complex empirical problems which have received undeservedly little attention. We have brought together these problems here, including some novel observations of our own.

We have argued that a significantly simpler analysis of FAs is possible if we simply assume that sentences can express assertions about event types, in addition to event tokens. This proposal, though to our knowledge novel, is in fact entirely natural in an ontology that includes events on the one hand and a generalized typetoken sortal distinction, on the other. We have also made explicit the important insight that some kind descriptions might impose the requirement that they be realized not simply by one instance but rather by a set of individual instances: this is, we claim, the requirement that FAs contribute. Both of these proposals suggest that the use natural language makes of the type-token distinction is significantly richer than it appears at first glance.

\section{References}

Asher, Nicholas: 2007, 'A Web of Words: Lexical Semantics in Context'. Ms., U. Texas Austin/CNRS.

Barwise, Jon and John Perry: 1983, Situations and Attitudes. MIT Press, Cambridge, MA.

Bolinger, Dwight: 1967, 'Adjectives in English: Attribution and predication', Linguа 18, 1-34.

Carlson, Greg: 1977, Reference to Kinds in English, Doctoral Dissertation, U. Mass. Amherst. 
Davidson, Donald: 1967, 'The logical form of action sentences', in N. Rescher (ed.), The Logic of Decision and Action, 81-95. University of Pittsburgh Press, Pittsburgh.

Ginzburg, Jonathan: 2005, 'Situation Semantics: The ontological balance sheet', Research on Language and Computation 3.4, 363-389.

Heim, Irene: 1982, The Semantics of Definite and Indefinite Noun Phrases, Doctoral Dissertation, University of Massachusetts at Amherst.

Kamp, Hans: 1981, 'A Theory of Truth and Semantic Representation', in J. Groenendijk, T. Janssen, and M. Stokhof (eds.), Formal Methods in the Study of Language, Vol. 1, 277-322. Mathematisch Centrum, Amsterdam.

Landman, Meredith and Marcin Morzycki: 2003, 'Event-Kinds and Manner Modification', in N. M. Antrim, G. Goodall, M. Schulte-Nafeh, and V. Samiian (eds.), Proceedings of the Western Conference in Linguistics (WECOL) 2002. California State University, Fresno.

Larson, Richard: 1998, 'Events and Modification in Nominals', in D. Strolovitch and A. Lawson (eds.), Proceedings from SALT VIII, 145-168. CLC Publication, Ithaca, NY.

McNally, Louise and Gemma Boleda: 2004, 'Relational Adjectives as Properties of Kinds', in O. Bonami and P. C. Hofherr (eds.), Empirical Issues in Syntax and Semantics, Vol. 5, 179-196. http://www.cssp.cnrs.fr/eiss5.

Moltmann, Friederike: 1997, Parts and Wholes in Semantics. Oxford University Press, Oxford.

Müller-Reichau, Olav: 2006, Sorting the World: On the Relevance of the KindLevel/Object-Level Distinction to Referential Semantics, Doctoral Dissertation, Universität Leipzig.

Parsons, Terence: 1990, Events in the Semantics of English: A Study in Subatomic Semantics, Current Studies in Linguistics Series 19. MIT Press, Cambridge, MA.

Reichenbach, Hans: 1947, Elements of Symbolic Logic. MacMillan, London.

Schäfer, Roland: 2007, 'On frequency adjectives', in E. Puig Waldmüller (ed.), Proceedings of Sinn und Bedeutung 11, 555-567. Universitat Pompeu Fabra, Barcelona.

Stump, Gregory T.: 1981, 'The interpretation of frequency adjectives', Linguistics and Philosophy 4, 221-257.

Zimmermann, Malte: 2003, 'Pluractionality and complex quantifier formation', Natural Language Semantics 11, 249-287. 